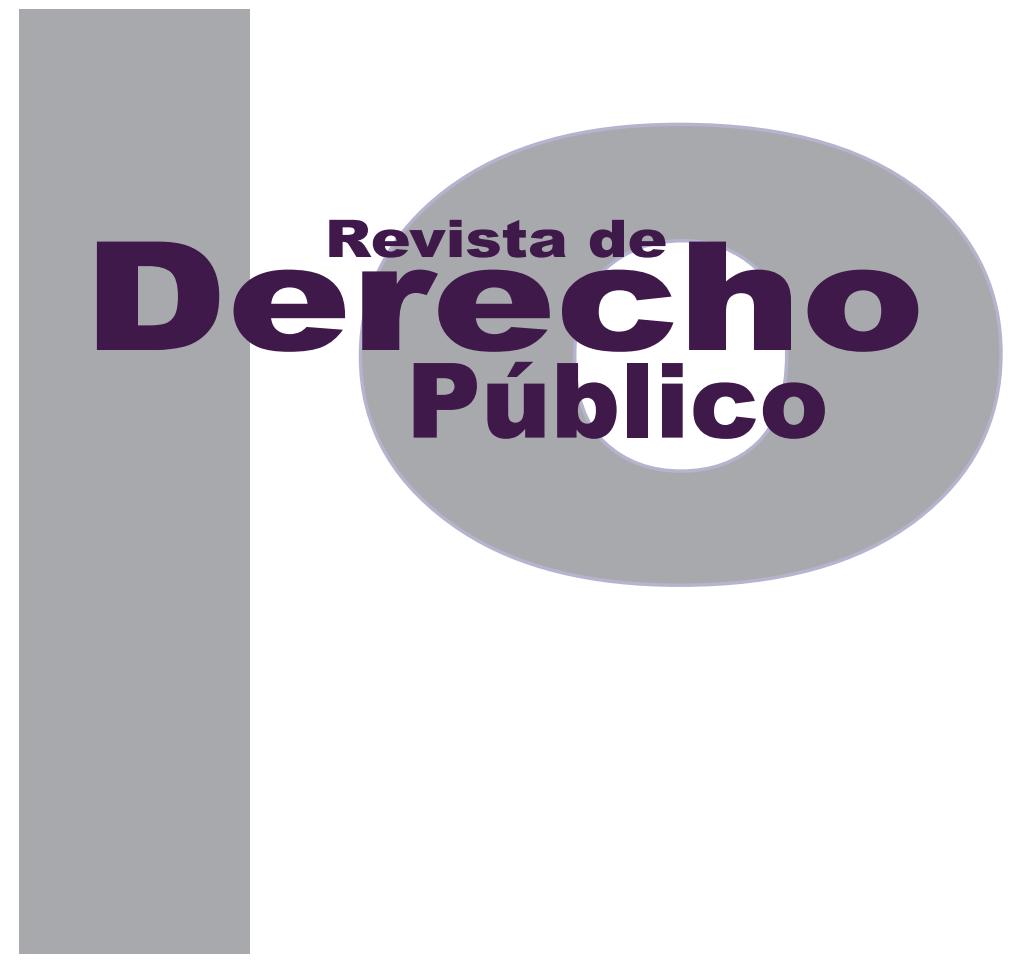

\title{
CONSTRUCCIONES SOCIALES, ÉTICA MÍNIMA Y PREJUICIOS: EL CUERPO DE LA MUJER EN LA MODERNIDAD
}

\author{
MARIANA GARCÍA JIMENO
}

Universidad de los Andes

Facultad de Derecho

Revista de Derecho Público N. ${ }^{\circ} 32$

Enero - Junio de 2014. ISSN 1909-7778 


\title{
Construcciones sociales, ética mínima y prejuicios: el cuerpo de la mujer en la modernidad*
}

\author{
Mariana García Jimeno**
}

ABSTRACT

\begin{abstract}
RESUMEN
En las sociedades modernas, los estereotipos de género han tenido como consecuencia la adjudicación de roles a los cuerpos de mujer y a los cuerpos de hombre, y las mismas normas legales no solo los han permito o reproducido sino, en muchos casos, creado. La literatura feminista ha descrito cómo, gracias a estas representaciones, el cuerpo de la mujer ha estado históricamente terrorizado, maternalizado y sexualizado. Este texto se ocupa de ejemplificar estos tres escenarios de acuerdo con las leyes colombianas actuales que reproducen esos roles de género dentro de nuestra sociedad y en entender estos escenarios como dilemas éticos que han sido invisibilizados.
\end{abstract}

PALABRAS clave: género, cuerpo, mujer, roles, ética, derecho.
Gender stereotypes have resulted in modern societies, in the allocation of roles to women's and men's bodies. In this sense, legal standards allow or reproduced and in many cases created these roles. The feminist literature has described how these roles of the female body has historically been terrorized, maternalized and sexualized. Thus, this text occupies on exemplifying this three scenarios in accordance with Colombian law that reproduce this gender roles inside our society and how to understood this scenarios as ethical dilemmas which had been invisible.

KEY WORDS: Gender, body, women, roles, ethic, law.

Cómo citar este artículo: García Jimeno, M. (Junio, 2014). Construcciones sociales, ética mínima y prejuicios: el cuerpo de la mujer en la modernidad. Revista de Derecho Público, 32.

* Estudiante de noveno semestre de Derecho en la Universidad de los Andes. Coordinadora del Doctorado en Derecho de la misma Universidad. 


\section{SUMARIO}

Introducción - I. ¿QUÉ ES LA ÉTICA? - II. EL CUERPO DE LA MUJER EN LA TEORÍA FEMINISTA - III. EL CUERPO Y LA LEY - A. La violencia intrafamiliar - B. La maternidad en las leyes laborales - C. La pornografía - IV. CONCLUSIONES - Referencias. 


\section{Introducción}

Mucho se ha dicho en la academia jurídica sobre cómo el derecho y la sociedad han construido socialmente lo que es y debe ser un cuerpo de mujer ${ }^{1}$, y de este modo se le han adjudicado ciertos roles que, al transgredirse, lo convierten en sujeto de veto y, sin duda, de exclusión. Teniendo en cuenta lo anterior, este texto busca explicar las construcciones sociales que el cuerpo de una mujer está obligado a representar, así como mostrar la forma en que, detrás de estos moldes creados socialmente, se invisibilizan dilemas éticos sobre estereotipos de género" ${ }^{2}$.

Para proceder en este sentido se desarrollan tres partes. En primer lugar, cuáles son las construcciones sociales que se han hecho del cuerpo de las mujeres a la luz de la teoría feminista moderna. En segundo lugar, se explica brevemente el concepto de ética que adopta este texto. Por último, a partir de tres casos específicos se explicará cómo esa construcción social sobre el cuerpo de la mujer, se traduce diariamente en problemas de carácter ético.

1 Nótese que no hablo de cuerpo femenino porque esta expresión ya incluye esa construcción de que el cuerpo de mujer necesariamente debe representar lo femenino y el cuerpo de hombre necesariamente lo masculino. La intención con este texto es, precisamente, romper con esa conexión que hasta el momento se ha mostrado inescindible.

2 Entendido como un "sistema de reglas, convenciones, normas sociales y prácticas institucionales que producen performativamente el sujeto que pretenden describir". Preciado, Beatriz. Testo Yonqui. Barcelona: Editorial Espasa, 2013, p. 81.

\section{I. ¿QUÉ ES LA ÉTICA?}

Como se planteó anteriormente, es de gran importancia definir qué significa la ética, primero, porque es la piedra angular de este texto; segundo, porque de acuerdo con la tesis de Tugendhat, la validez del proceso de clarificación de una palabra no está en su significado sino en el uso que se le dé a esta ${ }^{3}$. Así, en este texto, el término ética se entiende como un conjunto de imperativos mínimos que deben imponerse a sí mismas las personas, pero que en ninguna parte pueden confundirse con normas de tipo religioso o legal. La ética debe ser, entonces, un conjunto de creencias en las que existen dos mínimos morales, entendidos como "[p]rincipios, valores, actitudes o hábitos a los que no podemos renunciar sin renunciar a la vez a la propia humanidad"4. Estos dos mínimos son: el respeto por el otro y la igualdad de todos los seres humanos. Ahora, un problema o dilema ético surge en el momento en que estos dos mínimos, que denominaré principios éticos, se ponen en peligro al contrastarse con comportamientos que parecen normales en la modernidad pero que los desafían incuestionablemente. Dichos principios éticos no tienen otro propósito que lograr que cada persona tome las decisiones que considere necesarias para alcanzar la felicidad y la realización personal. Lo anterior no solo en el sentido de "aumenta[r] el placer

\footnotetext{
3 URIBE, Ángela. ¿En qué consiste una moral moderna? En: Hoyos, Guillermo y URIBE, Ángela. Convergencia entre ética y política. Bogotá: Siglo del Hombre Editores, Convergencia, IAP, 1998. tica. Madrid: Editorial Tecnos, 1995, p. 28.
} 
y reduc[ir] el dolor" 5 sino en el entendido de que los intereses de todos son igualmente válidos $\mathrm{y}$, por lo tanto, las decisiones de vida de cada quien, mientras respeten y traten como iguales a los demás, deben ser totalmente válidas.

Se escogieron estos dos mínimos éticos y no otros porque, por un lado, en "sociedades que se entienden a sí mismas como modernas es claro que en incontables momentos de nuestra vida cotidiana somos testigos, si no víctimas de infinitas formas de irrespeto de unos hacía otros" . Por otro lado, la estructura de las sociedades modernas suponen formalmente por lo menos la igualdad en derechos de las personas, pero dicha formalidad se ha quedado en eso y en la realidad del día a día somos igualmente testigos y víctimas de actos de desigualdad. En ese sentido, la igualdad y el respeto, que por lo menos en el preámbulo de la Constitución colombiana han sido entendidas como la dignidad humana, son en términos de Tungendhat derechos generales, es decir, derechos que tenemos todos con todos, y que están constituidos en sus extremos por derechos y deberes correlativos ${ }^{7}$. Por lo tanto, Todos somos responsables de hacer cumplir estos derechos generales, que en el marco de este texto más que derechos, serán mínimos éticos.

\footnotetext{
5 Singer, Peter. Ética práctica. Cambridge: Cambridge University Press, 1995, p. 17.

6 URIBE, Ángela. Ob. cit. p. 123.

7 Ibíd.
}

\section{EL CUERPO DE LA MUJER EN LA TEORÍA FEMINISTA}

El feminismo de finales del siglo XX y comienzos del XXI se ha preocupado ampliamente por definir cómo el derecho y la sociedad le han otorgado un significado estático y no discutible al cuerpo de la mujer. Así, Mary Joe Frug, por ejemplo, ha hablado de la terrorización, maternalización y sexualización del cuerpo de la mujer a través, sobre todo, de la creación de reglas jurídicas que reproducen normas sociales o culturales ${ }^{8}$. Terrorización en el sentido de que el derecho ha creado normas que protegen inadecuadamente a la mujer del abuso físico y que la hacen buscar refugio ante la inseguridad. En consecuencia, el cuerpo de las mujeres se ha hecho vulnerable en cuanto a que no puede protegerse por sí mismo y debe buscar amparo en otras personas o instituciones. La maternalización se explica como la serie de disposiciones a través de las cuales el derecho obliga a la mujer a asumir el rol de madre o le impone ciertas obligaciones con sus hijos. Por último, la sexualización se traduce en la imposición de una conducta sexual establecida como correcta y en la legitimación de la erotización del cuerpo de la mujer.

Las tres concepciones están estrechamente relacionadas, es más, las unas dependen de las otras para subsistir. Así, el fin de establecer una única conducta aceptada de sexo es que esta

Ver, Comentario: un manifiesto jurídico feminista posmoderno (versión inconclusa). En: García Villegas, Mauricio; Jaramillo, Isabel Cristina y Restrepo, Esteban. Crítica jurídica. Teoría y sociología jurídica en los Estados Unidos. Bogotá: Universidad de los Andes, Universidad Nacional, 2005. 
conduzca a la reproducción humana y, por lo tanto, lleve a que la mujer asuma el rol de madre. Igualmente, el que la mujer esté constantemente aterrorizada del significado que tiene su cuerpo en la sociedad, la vuelve más vulnerable a la hora de negociar las condiciones de su maternidad y su sexualidad con su pareja e incluso la pone en una situación de subordinación dentro de la sociedad.

\section{EL CUERPO Y LA LEY}

En Colombia existen muchos ejemplos de cómo la sociedad moderna ha decidido moldear el cuerpo de la mujer en estos tres sentidos. Para empezar, las leyes le han impuesto cargas desproporcionadas a las mujeres con respecto a sus hijos. Por ejemplo, en programas como Familias en Acción ${ }^{9}$ y otras políticas públicas de bienestar, pueden ser sujeto de estas siempre y cuando tengan hijos y sean madres cabeza de familia. De esta forma, las normas interpretan sus necesidades y las relacionan estrechamente con labores domésticas y de cuidado. ${ }^{10}$ Por otro lado, las normas jurídicas se han preocupado por desaprobar comportamientos sexuales como la prostitución o la homosexualidad pero se han mantenido al margen de cuestiones como la pornografía.

9 Alviar, Helena y Jaramillo, Isabel Cristina. Las políticas de distribución pública de recursos en el neoliberalismo: ¿una trampa para las mujeres? En: Feminismo y crítica jurídica: el análisis distributivo como alternativa crítica al legalismo liberal. Bogotá: Siglo del Hombre Editores, Universidad de los Andes, 2012.

10 Fraser, Nancy. Women, Welfare, and the Politics of Need Interpretation. En: Bowie, Lee; Higginhs, Kathleen y Meredith, Michaels. Thirteen questions in ethics and social philosophy. Fort Worth, TX: Harcourt Brace College Publishers, 1998.
Ahora bien, este texto, en específico, se preocupa por tres aspectos esenciales de la vida de las mujeres, que al haber sido moldeados en virtud de normas sociales o legales han ocultado un problema ético, que como se dijo al inicio, se esconde detrás de los estereotipos de género. En primer lugar, el procedimiento para denunciar la violencia intrafamiliar ante las comisarias. En segundo lugar, las leyes laborales sobre maternidad y, por último, la pornografía.

\section{A. La violencia intrafamiliar}

El primer dilema ético que surge como consecuencia de los estereotipos de género es la justificación de la violencia intrafamiliar, en defensa de la unidad familiar. Al respecto, se ha documentado cómo los comisarios de familia, al recibir denuncias sobre violencia intrafamiliar contra mujeres, antes de ordenar el desalojo por parte del victimario o la relocalización de la víctima y sus hijos, ordenan terapia de familia o que víctima y victimario se acerquen juntos a las comisarias para dialogar sobre las causas de la violencia ${ }^{11}$. Esta actitud de los comisarios de familia favorece comportamientos que, en palabras de Frug, aterrorizan el cuerpo de las mujeres hasta el punto de que cuando encuentran tantos obstáculos para que atiendan sus denuncias $^{12}$, prefieren no hacerlo y la violencia

11 CÁceres Monroy, Cesar Alejandro. Una casa de varios pisos: el acceso a la justicia en casos de violencia intrafamiliar en una Comisaría de Familia de Bogotá. En: Revista de Derecho Público, enero-junio 2012, n. ${ }^{\circ} 28$.

12 Según Cáceres, los comisarios de familia no toman las medidas de protección a favor de las mujeres cuando reciben sus denuncias; además, cuando estas acuden a las comisarías a denunciar violencia intrafamiliar se encuentran con una serie de funcionarios que las 
se convierte en una constante en su vida diaria. En este sentido, las construcciones sociales de lo que debe ser una mujer, como cuidadora y temerosa al aislamiento ${ }^{13}$, favorecen estos comportamientos de los comisarios y, de esta forma, el problema ético se refleja al poner en tensión el valor de la familia y los principios éticos para mantener la unidad familiar. Es decir, se anteponen creencias culturales sobre imperativos que deben regir toda la sociedad, como es el respeto por el cuerpo y la mente de la mujer y la obligatoria protección hacía ella que debe derivar de los principios de igualdad y respeto.

\section{B. La maternidad en las leyes laborales}

El segundo dilema ético que surge como consecuencia del análisis que se ha hecho es la maternalización del cuerpo de la mujer, a través de las leyes laborales que han propugnado por proteger el rol de madre antes que buscar la igualdad en la participación del hombre y la mujer en la crianza de los hijos. Así, el derecho se ha empeñado en ponerle cargas al empleador a la hora de contratar a una mujer madre o potencial madre, que terminan por desfavorecer su entrada al mercado laboral. La imposición que le ha hecho la sociedad al cuerpo de la mujer como reproductor, ha permitido la desigualdad de condiciones entre el hombre y la mujer a la hora de aplicar a un trabajo. De esta forma, el

hacen contar una y otra vez sus historias antes de llegar al comisario, para terminar en que estos, en muchos casos, las disuadan de hacerlo. Esto hace que, con frecuencia, desistan de presentar la denuncia.

13 Jaramillo, Isabel Cristina. La crítica feminista al derecho. En: WeSt, Robin. Género y teoría del derecho. Bogotá: Ediciones Uniandes, Instituto Pensar, Siglo del Hombre Editores, 2000. dilema ético que surge al respecto queda en cabeza del empleador, quien debe poner por encima de cualquier interés de carácter económico el hecho de que al elegir entre una mujer y un hombre debe inclinarse por quien esté más capacitado. Sin embargo, el que a la mujer se le hayan impuesto más cargas relativas a la maternidad y la crianza de los hijos tiene un peso a la hora de decidir, con lo cual no solo el mínimo moral de la igualdad entre todos los seres humanos queda en entredicho, sino que lo que entra a definir la escogencia es el sexo, es decir, se prefiere el cuerpo de hombre sobre el de mujer porque a este la sociedad le ha impuesto muchas menos cargas que le permitirán realizar de mejor manera su trabajo ${ }^{14}$.

\section{La pornografía}

Este último dilema es quizás el más difícil de plantear en términos éticos. Para hacerlo más claro es necesaria una explicación previa sobre lo que ha significado el cuerpo de la mujer en el sexo. La academia jurídica ha discutido ampliamente sobre la subordinación que ha sufrido la mujer a través del sexo. "Lo que define a la mujer como tal es lo que excita a los hombres", ha expresado polémicamente Mackinnon ${ }^{15}$, pero lo

14 En el periódico El Tiempo del domingo 15 de septiembre de 2013 se publicó un artículo sobre Sheryl Sandberg, alta ejecutiva de Facebook, titulado "A las mujeres no nos animan a ser líderes". En él Sandberg asegura que lo que debe hacerse no es igualar las cargas en el hogar sino obtener una mayor flexibilidad laboral. Esto lo que demuestra es que las mismas mujeres han puesto por encima de cualquier imperativo de igualdad entre los sexos la construcción social de madres como mejores cuidadoras. Escobar ChavarRia, Paula. A las mujeres no nos animan a ser líderes. El Tiempo, 15 de septiembre de 2013.

15 Mackinnon, Chatarine. Feminismo, marxismo, método y Estado: una agenda para la teoría. En: Garcia Villegas, Mauricio; Jaramillo, Isabel Cristina y Restrepo, Esteban. Crítica jurídica: teoría y sociología ju- 
que debe entenderse de esta afirmación es que las mujeres se han construido alrededor de lo que el hombre desea de un cuerpo de mujer. En este sentido, diversos autores han sostenido que la pornografía solo reproduce patrones de lo que sexualmente es deseable de una mujer y lleva al extremo su cuerpo como un "objeto para ser visto y gozado por otro"16. Así las cosas, la pornografía juega un papel determinante a la hora de sexualizar el cuerpo de la mujer, puesto que no solo lo instrumentaliza como un objeto del placer sino que además lo subordina a ello. “[L]a pornografía asume que la dominación es resorte para el deseo femenino, aprueba el comportamiento sexual y lo considera una respuesta natural al deseo"17.

Por lo anterior, debe considerarse a la pornografía como un modelo que establece formas "discriminatorias de ver el cuerpo y la sexualidad"18 $y$, por consiguiente, propone un dilema claro entre la importancia que le ha dado la sociedad moderna al sexo y la desigualdad que este ha creado entre el cuerpo de mujeres y hombres, cuando ponderarlos ni siquiera debería ser necesario en una sociedad en la que la igualdad y el respeto por el otro son un mínimo moral. Cabe aclarar que la pornografía no es en sí misma la creadora de la desigualdad entre el cuerpo de

rídica en los Estados Unidos. Bogotá: Universidad de los Andes, Universidad Nacional, 2006.

16 Gómez, María Mercedes. Introducción. En: Mackinnon, Chatarine y Posner, Richard. Derecho y pornografía. Bogotá: Nuevo Pensamiento Jurídico, Siglo del Hombre Editores, Universidad de los Andes, 1997, p. 27.

17 Ibíd., p. 29.

18 Ibíd., p. 30 hombres y mujeres; solo está circunscrita a un contexto permanente y repetitivo de patrones de desigualdad que esta simplemente acrecienta.

Lo que es verdaderamente preocupante es que la legislación ha decidido abstenerse completamente de entrar a regular el mundo de la pornografía, excepto la infantil por el problema que ha representado para la sociedad, pero las políticas públicas nunca han pensado en esta como aliciente para el rol desigual de la mujer en la sociedad; tampoco se ha planteado el dilema de la discriminación que encierra y la ha dejado totalmente de lado en virtud de la definición liberal de la libertad de expresión.

Ahora bien, más allá de irrespetar esos mínimos morales, las consecuencias de la construcción del cuerpo de la mujer de acuerdo con estos estándares crea en la realidad un prejuicio contra aquellas que no llenan las expectativas de la sociedad. Las mujeres que dejan de lado el rol de madre para convertirse en exitosas trabajadoras, en el imaginario social no solo son vistas como seres incompletos dado que la maternidad es una faceta inseparable de su condición, sino que con frecuencia se les califica de mandonas y poco femeninas solo por el hecho de ejercer el liderazgo de una forma eficiente pero inusual $^{19}$.

\section{CONCLUSIONES}

No hay que ir muy lejos para encontrar que cada estereotipo es un prejuicio en sí mismo. El que

19 Escobar Chavarría, ob. cit. 
se le hayan asignado características a cada cuerpo, y que de eso se desprenda un determinado rol en la sociedad, es la mejor manera de prejuzgar. Definir una persona por sus genitales y no por sus características como individuo, y determinar las actitudes que debe adoptar por tener un determinado cuerpo no es otra cosa que la expresión más elemental de un prejuicio.

Parecería que la lucha por la igualdad de género es una simple cuestión de derechos; sin embargo, es un problema que va mucho más allá de definir unas prerrogativas a favor de la mujer en la ley: es la reivindicación de unos mínimos éticos de los que depende que se le reconozca como igual al hombre, que se le respete la decisión de vida que decida adoptar, que no se le impongan cargas que incluso pueden degradarla como ser humano. El que algún día el cuerpo de la mujer deje de ser un objeto para concretar algunos fines de la sociedad debe ser un imperativo de cualquier grupo humano que alegue estar regido por mínimos morales en su actuar.

\section{Referencias}

ALVIAR, Helena y JARAMILLO, Isabel Cristina. Las políticas de distribución pública de recursos en el neoliberalismo: ¿una trampa para las mujeres? En: Feminismo y crítica jurídica: el análisis distributivo como alternativa crítica al legalismo liberal. Bogotá: Siglo del Hombre Editores, Universidad de los Andes, 2012.

CÁCERES MONROY, Cesar Alejandro. Una casa de varios pisos: el acceso a la justicia en casos de violencia intrafamiliar en una Comisa- ría de Familia de Bogotá. En: Revista de Derecho Público, enero-junio, 2012, n. ${ }^{\circ} 28$.

CORTINA, Adela. Ética mínima: introducción a la filosofía práctica. Madrid: Editorial Tecnos, 1995.

ESCOBAR CHAVARRÍA, Paula. A las mujeres no nos animan a ser líderes. El Tiempo, 15 de septiembre de 2013.

FRASER, Nancy. Women, Welfare, and the Politics of Need Interpretation. En: BOWIE, Lee; HIGGINHS, Kathleen y MEREDITH, Michaels. Thirteen questions in ethics and social philosophy. Fort Worth, TX: Harcourt Brace College Publishers, 1998.

FRUG, Mary Joe. Comentario: un manifiesto jurídico feminista posmoderno (versión inconclusa). En: GARCÍA VILLEGAS, Mauricio; JARAMILLO, Isabel Cristina y RESTREPO, Esteban. Crítica jurídica: teoría y sociología jurídica en los Estados Unidos. Bogotá: Universidad de Ios Andes, Universidad Nacional, 2005.

GÓMEZ, María Mercedes. Introducción. En: MACKINNON, Chatarine y POSNER, Richard. Derecho y pornografía. Bogotá: Nuevo Pensamiento Jurídico, Siglo del Hombre Editores, Universidad de los Andes, 1997.

JARAMILLO, Isabel Cristina. La crítica feminista al derecho. En: WEST, Robin. Género y teoría del derecho. Bogotá: Ediciones Uniandes, Instituto Pensar, Siglo del Hombre Editores, 2000. 
MACKINNON, Chatarine. Feminismo, marxismo, método y Estado: una agenda para la teoría. En: GARCÍA VILLEGAS, Mauricio; JARAMILLO, Isabel Cristina y RESTREPO, Esteban. Crítica jurídica: teoría y sociología jurídica en los Estados Unidos. Bogotá: Universidad de los Andes, Universidad Nacional, 2006.

PRECIADO, Beatriz. Testo Yonqui. Barcelona:

Editorial Espasa, 2013.
SINGER, Peter. Ética práctica. Cambridge: Cambridge University Press, 1995.

URIBE, Ángela. ¿En qué consiste una moral moderna? En: HOYOS, Guillermo y URIBE, Ángela. Convergencia entre ética y política. Bogotá: Siglo del Hombre Editores, Convergencia, IAP, 1998. 